\title{
Tendencias en el conformado de suspensiones cerámicas
}

\author{
R. MORENO \\ Instituto de Cerámica y Vidrio, CSIC, 28500 Arganda del Rey, Madrid
}

\begin{abstract}
El procesamiento coloidal de materiales cerámicos permite mejorar las propiedades finales de los productos, reduciendo el número y tamaño de los defectos, y aumentando la reproducibilidad del proceso. Esto exige, por una parte, el adecuado conocimiento de los parámetros coloidequímicos y reológicos que rigen la estabilidad de la suspensión y, por otra, el control de las variables específicas de cada proceso de conformado. La técnica de colaje se ha utilizado tradicionalmente en la industria, dada su gran versatilidad, su bajo costo y la elevada uniformidad de las piezas así obtenidas. Sin embargo, el desarrollo industrial impone la búsqueda de nuevas técnicas de conformado capaces de aunar las ventajas del colaje (como la posibilidad de obtener piezas de forma casi final, con el fin de reducir o eliminar la etapa de mecanización) con las propias de otros procesos (como los de prensado), como son la reducción del ciclo de fabricación y la automatización del proceso. Esto ha impulsado el desarrollo de nuevos procesos de conformado en los que la manipulación de las propiedades coloidales de una suspensión permiten obtener piezas de forma casi neta. En este trabajo se muestran las bases de dichas técnicas y se discuten las tendencias actuales en el conformado coloidal de materiales cerámicos.
\end{abstract}

Palabras clave: Procesamiento, Conformado, Suspensiones

\section{Trends in slip forming of ceramics}

Colloidal processing of ceramics allows to improve the final properties of the products by reducing the number and size of defects, enhancing the reproducibility of the process. This needs on one hand an adequate knowledge of colloidal and rheological parameters that control the stability of the suspension and, on the other hand, the control of the specific parameters of each forming technique. Slip casting has been widely used in the industry due to its high versatility, the low cost and the high uniformity in the so cast bodies. However, the industrial development needs the search of new forming techniques capable to combine the advantages of casting processes (as the possibility to obtain near-net shaped parts where machining is reduced or even avoided) with those of other processes (such as pressing) mainly related to the reduction of the fabrication cycle and the possibility of automation. All this has favoured the development of new forming techniques where a proper manipulation of the colloidal properties of a slip makes possible to obtain near-net shaped parts. This work shows the basis of such processes and discusses the actual tendencies in colloidal forming of ceramics.

Key words: Processing, Forming, Slips

\section{INTRODUCCIÓN}

El procesamiento cerámico es el conjunto de operaciones por las que, a partir de uno o varios polvos de partida, se llega a obtener una pieza de la forma y estructura deseadas. La fabricación de un producto, pues, requiere el establecimiento de una sistemática que permita ejercer un adecuado control en cada una de las etapas del proceso, ya que las propiedades del sistema en cada etapa determinan todas las restantes (1-3). La secuencia típica de fabricación de un producto cerámico se muestra en la Figura 1. Muchos procesos de conformado utilizados en otras áreas, como son los métodos de deformación o de fusión comúnmente utilizados en metalurgia, no son aceptables en cerámica. En el procesamiento cerámico se parte de polvos, naturales o sintéticos, que normalmente precisan de una serie de tratamientos de adecuación o beneficio, para su consolidación. Los tratamientos a los que se somete el polvo dependen de la forma y tamaño de la pieza a obtener y de la uniformidad microestructural deseada. Tras la consolidación, la pieza en verde se somete a un ciclo térmico, tras el cual adquiere sus propiedades finales. Durante cada una de estas etapas se pueden introducir defectos o heterogeneidades que persistirán o darán lugar a nuevas inhomogeneidades durante la etapa de densificación y desarrollo microestructural.

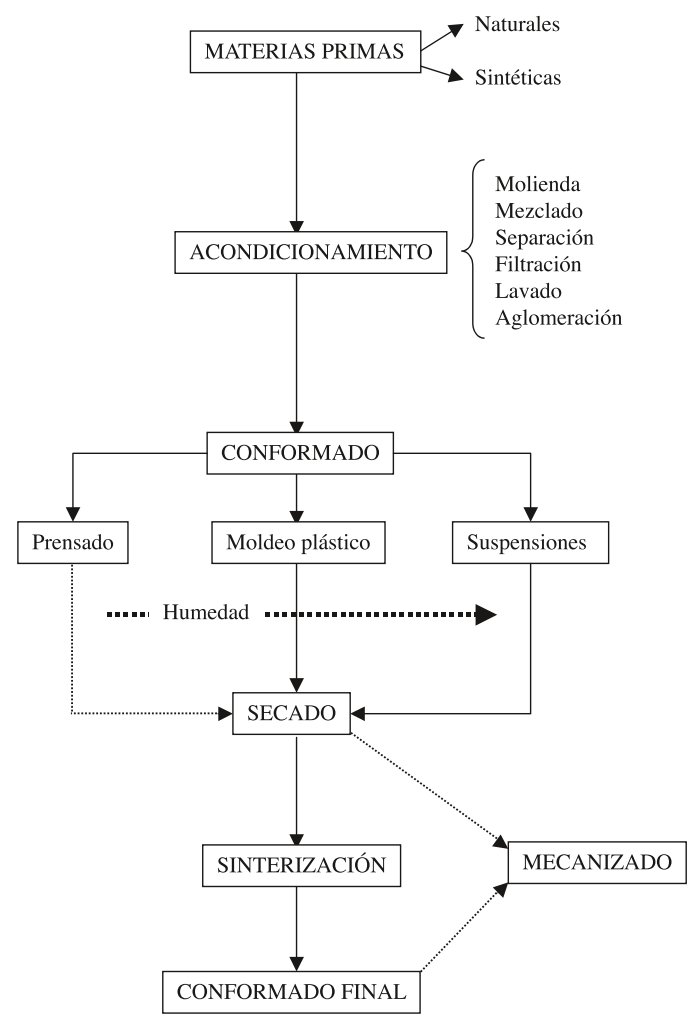

Fig. 1.- Esquema de la estapas típicas del procesamiento cerámico. 
El objetivo básico del procesamiento cerámico es profundizar en el control de los parámetros fisicoquímicos involucrados en cada una de las etapas del proceso con el fín de reducir en lo posible la presencia de defectos (4).

El control de los defectos para la obtención de un buen material ha obligado a profundizar en dos líneas de actuación: los polvos de partida y el procesamiento de dichos polvos. En la década de los 80 empezó a admitirse la importancia del procesamiento de polvos en las propiedades finales del producto (5-7). Actualmente, el desarrollo de nuevas rutas de obtención de polvos de mayor pureza y homogeneidad y menor tamaño constituye una de las líneas de investigación prioritarias en el área de los materiales cerámicos. Evidentemente, un mal procesado de estos polvos introducirá nuevos defectos y, por tanto, menores prestaciones en el producto final. De esta forma, se ha hecho necesario el desarrollo de una metodología capaz de optimizar el procesado de esos polvos sin introducir nuevos defectos. Para ello es imprescindible conocer el estado superficial de las partículas y controlarlo con el fín de evitar los fenómenos de aglomeración, que darán lugar e heterogeneidades y peores prestaciones en la pieza final.

Por otra parte, en las modernas tecnologías se utilizan componentes de diversos materiales. Así, un componente cerámico no es un ente aislado, sino que debe cubrir unas expectativas en servicio, por lo que su selección debe venir definida por el uso y destino que se le va a dar en función de sus propiedades, así como de la geometría adecuada para su correcto funcionamiento $(8,9)$.

Todo ello ha contribuido a impulsar la búsqueda de técnicas de conformado que permitan obtener materiales de mayor vida media y mejores prestaciones, así como una mayor reproducibilidad en su obtención.

\section{CRITERIOS PARA EL CONFORMADO}

La selección de un método de conformado para la fabricación de un producto cerámico depende de diversos factores, como son la geometría de la pieza, la cantidad de piezas a producir y la homogeneidad y reproducibilidad de las piezas conformadas (3,10-12). Su implantación en planta exigirá, además, que sea rentable y no contaminante. La rentabilidad a su vez, depende de las inversiones necesarias en costos de producción, instalaciones y equipamiento, precio y cantidad de aditivos de procesamiento, etc. Pero la etapa más costosa en la fabricación de componentes cerámicos es, posiblemente, la de mecanizado del material sinterizado para proporcionar la forma definitiva en su aplicación. El desarrollo de nuevos materiales se ha centrado en mejorar sus propiedades, pero no se ha dedicado un esfuerzo paralelo para facilitar el mecanizado. Dada la extrema dureza e inherente fragilidad de los materiales cerámicos, su mecanizado es complejo, haciendose necesario el uso de diamante sinterizado. Otras técnicas de mecanizado más recientes están adquiriendo un papel relevante en la práctica industrial, como es el caso de las técnicas láser y ultrasónicas.

Por lo tanto, uno de los objetivos fundamentales de la ciencia del procesamiento actual es encontrar métodos que reúnan todas las ventajas anteriores y permitan la obtención de piezas de forma definitiva (en inglés, near-net shaping) (13). La figura 2 esquematiza los criterios a tener en cuenta para la selección de un proceso de conformado.

Desgraciadamente, ningún método es adecuado para todas las necesidades, por lo que la selección del método dependerá de todos los parámetros anteriores.

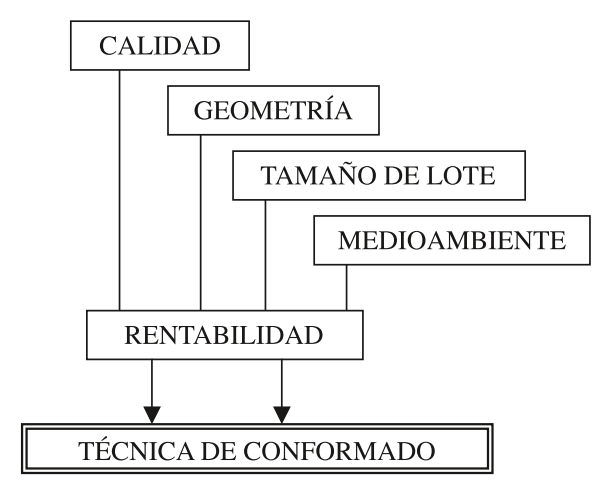

Fig. 2.- Criterios para la selección de una técnica de conformado.

Todo ello ha impulsado en las dos últimas décadas el desarrollo de métodos coloidales para el conformado. Estos han demostrado su eficacia para obtener materiales de forma compleja, mayor densidad y uniformidad y menor costo.

\section{EL PROCESAMIENTO COLOIDAL}

En los años 80 el procesamiento cerámico experimentó un tremendo auge como consecuencia de la reivindicación de ciertos investigadores $(14,15)$ de darle una perspectiva científica que complementara la ingenieril que tenía hasta entonces. Esto hizo comprender la importancia de los sistemas partículados y de sus propiedades fisicoquímicas, lo que permitió la implantación de otras ciencias en el estudio de los materiales cerámicos. Un caso significativo de incorporación de otras ciencias al procesamiento lo constituye la ciencia de superficies y coloides. La implantación de una sistemática científica, más preocupada por el entendimiento de los procesos fisicoquímicos involucrados en el proceso y el control de los mecanismos de estabilidad de las suspensiones, fué desplazando a los métodos tradicionales de prueba y error. De esta forma, se popularizó el término procesamiento coloidal para referirse a las técnicas de conformado que hacían uso de suspensiones. Mediante el control de las fuerzas de interacción desarrolladas entre las partículas en suspensión, se ha logrado mejorar notablemente la uniformidad del producto sinterizado y, por tanto, sus propiedades de comportamiento (16-19). El control de la estabilidad y homogeneidad de una suspensión para su posterior conformado ha sido posible gracias a la incorporación de otra ciencia, en este caso la reología. El desarrollo actual de nuevos modos de operación más precisos, como son los de esfuerzo controlado o las medidas en oscilación forzada, ha permitido conocer mucho mejor las características de flujo de la suspensión en distintas condiciones, así como el alcance de las fuerzas de interacción entre partículas en las propiedades de flujo (20-22).

En todos los procesos coloidales de conformado se parte de un denominador común: una suspensión estable y homogénea, cuya preparación exige el conocimiento previo de las distintas fuerzas de interacción que se pueden originar en el seno de la misma. Una suspensión no puede considerarse simplemente como un conjunto de dos partículas que interaccionan, sino la combinación de muchos cuerpos que pueden interaccionar. Esto dificulta aún más el conocimiento de la coloidequímica de suspensiones concentradas. Además de las posibles interacciones entre partículas, existen también interacciones partículadisolvente y disolvente-disolvente. Estas interacciones, poco consideradas tradicionalmente, han permitido en los últimos 
años el desarrollo de nuevos métodos de conformado basados en las interacciones de corto alcance (23-24).

El mayor problema a la hora de preparar una suspensión concentrada es mantener las partículas dispersas en el seno del líquido debido a la tendencia a unirse entre sí mediante fuerzas de tipo London-van der Waals, que se originan como consecuencia de la formación de dipolos instantáneos a partir de fluctuaciones en la distribución de carga electrónica. Estas fuerzas de atracción están siempre presentes y son excepcionalmente intensas a distancias de separación entre partículas cercanas a la de contacto.

La estabilidad de una suspensión solo se logra cuando se desarrollan fuerzas de repulsión que dominan sobre las de atraccción. Esto se puede conseguir de varias formas. El caso más sencillo es el postulado por la teoría de Derjaguin-LandauVerwey-Overbeek (DLVO), según la cual el potencial total de interacción viene dado por la suma directa del potencial de atracción de London y del potencial de repulsión electrostático. Este último es el que se desarrolla como consecuencia de la interacción entre las dobles capas que se forman alrededor de las partículas inmersas en un líquido polar. También se puede alcanzar una buena estabilidad mediante el mecanismo estérico, en el que la presencia de polímeros adsorbidos sobre la superficie de las partículas impide su coagulación. Si los polímeros adsorbidos están cargados (polielectrolitos) se alcanza la estabilidad mediante un mecanismo electrostérico, en el que a distancias cortas los polímeros impiden el contacto, mientras que a distancias largas es la carga asociada a aquellos la que proporciona la estabilidad electrostática. Existen numerosos libros y revisiones sobre los mecanismos de estabilidad de suspensiones (25-29), que no son el objetivo de este trabajo. En adelante, se considerarán los parámetros asociados al procesamiento asumiendo que se parte de una suspensión estable y homogénea.

\section{LA CONSOLIDACIÓN}

\subsection{Esquema de las técnicas de conformado.}

En cualquier técnica de conformado a partir de suspensiones se parte de la preparación de una suspensión estable y homogénea, por lo que la etapa de molienda y mezclado es crítica para que las partículas alcancen un equilibrio superficial (30). Además, se precisa alcanzar una elevada concentración en sólidos, con el fín de reducir la cantidad de líquido a eliminar durante el secado, pero sin dificultar el comportamiento de flujo, ya que esto impediría el llenado por vertido o inyección en moldes y cavidades. Si la suspensión es estable, las fuerzas de repulsión impedirán la tendencia de las partículas a atraerse y, por tanto, se evitará la presencia de aglomerados. Esto es lo que confiere al procesamiento coloidal la capacidad de reducir el número y tamaño de defectos y dar lugar a una microestructura más fina y uniforme.

El conformado supone la consolidación de las partículas de la suspensión para formar un compacto tras la eliminación del líquido. Según esta definición se podrían clasificar los métodos de conformado coloidal en función del mecanismo de separación sólido/líquido. Así, en las técnicas de filtración o colaje, la consolidación se logra por filtración del líquido en un molde poroso (31-34). En el colaje en cinta la suspensión vertida sobre un sustrato no poroso se compacta por simple evaporación del disolvente $(35,36)$, mientras que en la deposición electroforética el mecanismo dominante es la migración de partículas y su posterior secado en el electrodo $(37,38)$. Otras tendencias más recientes se basan en la coagulación/floculación de una suspensión previamente estabilizada, con el consiguiente aumento de viscosidad que permite retener la estructura (39). Asimismo, han cobrado auge las técnicas de consolidación por gelificación/polimerización, bien por adición de aditivos que gelifican o de monómeros que polimerizan durante la consolidación. La Tabla 1 resume los procesos de conformado a partir de suspensiones más conocidos.

TAbla 1. Proceso de CONFORMAdo A PARTIR DE SUSPENSIONES.

\begin{tabular}{|c|c|c|c|}
\hline FILTRACIÓN & $\begin{array}{l}\text { DEPOSICIÓN } \\
\text { EVAPORACIÓN }\end{array}$ & $\begin{array}{l}\text { FLOCULACIÓN } \\
\text { COAGULACIÓN }\end{array}$ & GELIFICACIÓN \\
\hline Colaje & Colaje en cinta & Fuerzas corto alcance & $\begin{array}{l}\text { Polimerización } \\
\text { (Gelcasting) }\end{array}$ \\
\hline Colaje con presión & Electroforesis & Puentes poliméricos (TIF) & $\begin{array}{l}\text { Moldeo por } \\
\text { inyección }\end{array}$ \\
\hline $\begin{array}{l}\text { Colaje con vacío } \\
\text { Colaje } \\
\text { con micrrondas }\end{array}$ & CVD, PVD... & Coagulación directa (DCC) & $\begin{array}{l}\text {-En frío } \\
\text { (agar, agarosa) } \\
\text {-En caliente } \\
\text { (metilcelulosas) }\end{array}$ \\
\hline
\end{tabular}

Se podría establecer otra posible clasificación en función de la forma a reproducir. En este caso, habría que distinguir entre el conformado de sustratos, capas y recubrimientos (colaje en cinta, electroforesis, inmersión, etc) o piezas de geometría compleja, que a su vez podrían ser huecas (métodos de colaje) o macizas (colaje con presión, procesos de gelificación y coagulación/floculación).

Estas técnicas de conformado han dado resultados muy positivos, por lo que es de prever su creciente incorporación en las tecnologías de producción previstas a corto plazo. Por una parte, la sociedad está cada vez más concienciada de la imperiosa necesidad de proteger el medio ambiente, por lo que la sustitución de disolventes orgánicos por agua es fundamental para la implantación industrial de nuevos procesos. Junto a la incidencia medioambiental, deben sumarse la incidencia del uso de agua en mejorar las condiciones de trabajo. Otra razón poderosa para desarrollar nuevos procesos en agua es el elevado coste de los aditivos orgánicos que, en definitiva, se utilizan solo como vehículo soporte y luego se eliminan.

El otro aspecto destacado de las técnicas coloidales es la posibilidad de reproducir una gran variedad de formas, en algunos casos de gran complejidad. Uno de los retos más importantes de la fabricación es establecer nuevas rutas simples y viables para la obtención de piezas de forma casi definitiva, de forma que se pueda reducir, e incluso eliminar, la etapa de mecanizado.

\subsection{Métodos de filtración.}

De todas las técnicas de conformado a partir de suspensiones, la más empleada y mejor conocida es la de colaje en molde permeable (slip casting) (31-34,40). Este proceso presenta importantes ventajas con respecto a los métodos de prensado, extrusión, etc, ya que, además de conducir a mayor homogeneidad microestructural, permite obtener piezas de simetría compleja y forma casi definitiva, reduciendo el mecanizado. Además, normalmente se realiza en agua, con las ventajas que ello conlleva, y no requiere grandes inversiones en equipamiento.

Sin embargo, la técnica de colaje presenta importantes limitaciones que dificultan su implantación en la fabricación de lotes grandes de piezas $(41,42)$. En este sentido, cabe destacar los siguientes aspectos: 
- Tiempo. Dada la cinética del proceso, en la que el espesor de pared formada es proporcional a la raiz cuadrada del tiempo de colaje, el máximo espesor está limitado a unos pocos milímetros. Mayores espesores requieren tiempos desproporcionadamente largos en los que pueden producirse fenómenos de sedimentación y desestabilización de la suspensión.

- Desmoldeo. Una vez alcanzado el espesor deseado, el desmoldeo requiere la filtración de $\sim 15 \%$ de agua, lo que supone tiempos de residencia de, incluso, varias horas.

- Espacio. A menudo los moldes son pesados y voluminosos. Como el tiempo de succión es alto se precisa un elevado número de moldes para no frenar la producción.

- Trabajo. Los moldes de escayola se deterioran tras un cierto número de coladas, que oscilan entre 10 y poco más de 100 , dependiendo del material, de la agresividad del medio y de la precisión superficial deseada en la pieza colada. La reposición contínua de moldes es vital para la continuidad de la producción.

- Almacenamiento. Los cambios de forma de modelos exigen espacio donde depositarlos, además de conservar los previamente existentes.

- Especialización. El diseño de moldes y el control de las propiedades de la suspensión y de las condiciones de filtración exigen personal especializado.

- Control reológico. La suspensión se ve afectada por el envejecimiento, la presencia de impurezas, la contaminación por deterioro del molde de escayola, etc., lo cual requiere el control reológico de la suspensión con el fín de asegurar la homogeneidad de la misma, previniendo fenómenos de sedimentación.

- Automatización. Dada la dificultad de ordenar los lotes de producción en las distintas etapas del proceso resulta enormemente compleja la automatización global del mismo.

Todos estos inconvenientes técnicos han impulsado el desarrollo de técnicas alternativas al colaje en las que, mediante un factor externo, se acelere el proceso de drenaje. Los métodos más clásicos son la aplicación de vacío (1-3), de presión (41-48), de centrifugación $(49,50)$ o de una fuente de microondas $(51,52)$.

De todos ellos, el que más expectativas ha suscitado ha sido el de colaje con presión. Este proceso, aparecido en los años 70, empezó a ser explotado industrialmente hacia 1985 y ya en 1990 se erigía en una técnica firmemente establecida en la fabricación de cerámica blanca y sanitarios. Este desarrollo vertiginoso demuestra la tendencia de los últimos años a desarrollar métodos avanzados en materiales considerados tradicionales. Las mayores ventajas del proceso de colaje con presión se resumen en los siguientes aspectos: 1) Aceleración drástica del ciclo de producción. La cinética es, al menos, un orden de magnitud superior a la del colaje convencional, 2) el proceso es fácilmente automatizable, 3) permite obtener piezas de forma compleja casi final, 4) se pueden obtener piezas de gran tamaño y, a diferencia de lo que ocurre en colaje, gran espesor, y 5) la uniformidad de la pieza es alta (aunque las densidades en verde son ligeramente inferiores a las obtenidas por colaje). Todas estas ventajas han hecho posible que en la actualidad funcionen plantas en bateria con importantes velocidades de producción. Se estima que en una planta en bateria se pueden utilizar hasta 12 moldes. Para un valor medio de 6 moldes el tiempo típico de obtención de la pieza en verde es de unos 15 minutos. En el caso concreto de sanitarios, el llenado de los 6 moldes requiere unos 150-180 1 de suspensión y el tiempo necesario para la sustitución de los moldes es de 30-60 minutos. En la Tabla 2 se muestra un ejemplo del tiempo empleado normalmente en cada una de las operaciones del proceso de conformado por colaje con presión de sanitarios. En cerámica de mesa la producción es mucho más rápida, ya que el drenaje es más sencillo, por lo que el tiempo de operación se puede reducir a unos 2-3 minutos.

No obstante, esta técnica no está aún suficientemente desarrollada, especialmente para el conformado de cerámica técnicas. En general, los materiales cerámicos avanzados parten de polvos submicrónicos, cuyo empaquetamiento requiere la aplicación de mayores presiones (del orden de varios $\mathrm{MPa})(53,54)$. Esto implica necesariamente el rediseño de las máquinas de prensado de forma que permitan aumentar el rango de presiones, lo cual no constituye un problema técnico importante en sí mismo. Sin embargo, el uso de mayores presiones hace necesa-

TABlA 2. CiClOS DE TIEMPO TíPICOS EN LA FABRICACIÓN DE SANITARIOS POR COLAJE CON PRESIÓN.

PROCESO $\operatorname{TIEMPO}(\mathrm{s})$

$\begin{array}{cc}\text { Cerrado de moldes } & 20 \\ \text { Llenado de moldes } & 110 \\ \text { Tiempo de prensado } & 660 \\ \text { Descompresión } & 25 \\ \text { Postcompresión } & 110 \\ \text { Apertura de moldes } & 10 \\ \text { Tiempo total } & 935\end{array}$

ria la sustitución de los materiales empleados en la confección de moldes. Evidentemente, la escayola queda descartada, dada su pobre resistencia mecánica. La escasa información disponible sobre estos materiales constituye una de las más serias trabas actuales para el desarrollo del colaje con presión. Por otra parte, el propio caracter submicrónico de los polvos de partida pueden dificultar la cinética de filtración, e incluso la uniformidad de la pieza, dado el posible efecto de taponamiento que producen las partículas más finas al migrar y quedar atrapadas en los canales que dejan las partículas gruesas (55). Pese a ello, la enorme versatilidad del proceso y su gran capacidad para la producción de grandes lotes hacen de la técnica de colaje con presión una de las más esperanzadoras rutas para el conformado neto de piezas cerámicas. Para ilustrar la enorme repercusión de la presión en la cinética de filtración, la figura 3 muestra las curvas de formación de pared de una misma suspensión colada en molde de escayola y en una prensa al aplicar una presión de 6,8 $\mathrm{MPa}(56)$.

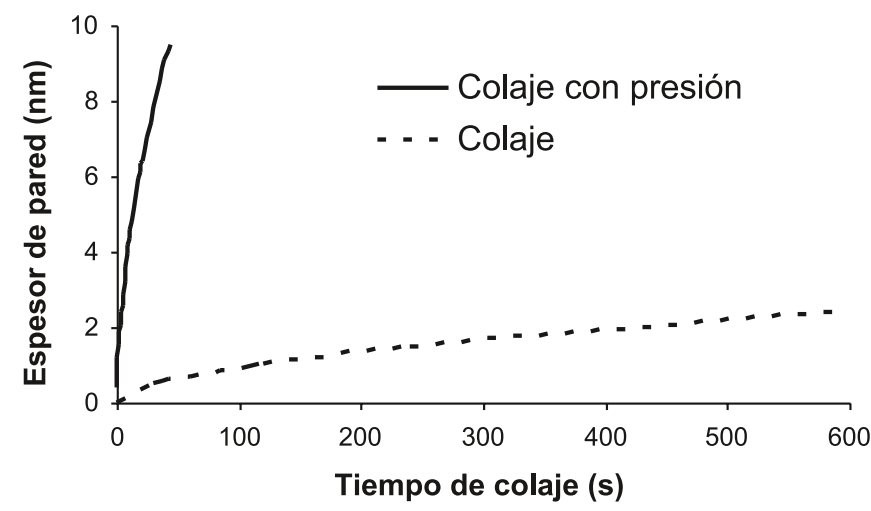

Fig. 3.- Curvas de colaje y colaje con presión (a 6,8 $\mathrm{MPa}$ ) para una suspensión al $60 \%$ sólidos de $\mathrm{SiC}$ con $0,5 \%$ de boro amorfo y $3 \%$ de negro de humo. 


\subsection{Métodos de floculación/coagulación.}

En los procesos de colaje se parte de suspensiones de baja viscosidad, en los que la propia fluidez puede afectar a la homogeneidad del empaquetamiento, ya que se pueden originar campos de flujo y de tensiones. Esto ha llevado a algunos investigadores a idear nuevos procesos en los que se produzca un aumento de viscosidad durante la consolidación, de forma que se reduzcan los posibles defectos asociados a la fluidez de la suspensión.

Una de las formas de manipular una suspensión es favorecer la atracción entre partículas para permitir la creación de una red estructural. Aplicando los principios de la coloidequímica, esto se puede hacer considerando el potencial de interacción entre partículas en función de la distancia de separación. En una suspensión estabilizada electrostáticamente, se puede superar la barrera de energía de repulsión forzando a las partículas a caer en un pozo atractivo de potencial, el cual puede corresponder a un mínimo primario (en el que las partículas entran en contacto directo y se produce coagulación) o secundario (en el que se produce la floculación por atracción entre partículas que nunca llegan a tocarse). La figura 4 ilustra los distintos estados de estabilidad de una suspensión en la curva de potencial de interacción en función de la distancia, según describe la teoría de DLVO para el mecanismo electrostático de estabilización. Una primera posibilidad para crear una red atractiva es partir de una suspensión defloculada y llevarla posteriormente al $\mathrm{pH}$ correspondiente al punto isoeléctrico, con lo que se produce un aumento brusco de viscosidad que permite consolidar las partículas. Esta es la base del proceso de coagulación directa (direct coagulation casting, DCC) desarrollado por Gauckler et al. $(57,58)$ Además del cambio de $\mathrm{pH}$ hacia el isoeléctrico, dichos autores han favorecido la consolidación provocando la desestabilización interna debida a la formación de sales. Así, proponen el uso de urea que, por acción enzimática de la ureasa, se descompone en una sal de carbonato amónico. De esta forma, se consigue añadir una concentración de sal de hasta $2 \mathrm{M}$, que provoca la coagulación. En este proceso se usan suspensiones con concentraciones en sólidos de 50-60\% volumen, con tiempos de 10-90 minutos y temperaturas de coagulación en torno a los $70^{\circ} \mathrm{C}$. El bajo contenido en orgánicos $(<1 \%$ vol $)$ permite la obtención de piezas complejas a bajo coste sin ciclo de extracción de orgánicos, densidades elevadas y microestructuras homogéneas.

La figura 5 ilustra la variación que experimentan las partículas en su estabilidad al cambiar el $\mathrm{pH}$ hasta un valor cercano al punto isoeléctrico, donde el potencial zeta es nulo (figura 5.a) y las partículas caen en un pozo atractivo de potencial correspondiente a un mínimo primario (figura 5.b), en el cual se produce la coagulación.

Otra forma de producir la red atractiva de partículas es forzar la floculación en mínimo secundario mediante la adición de sales a una suspensión defloculada sin cambiar el pH. Las suspensiones así preparadas tenían una viscosidad de al menos un orden de magnitud inferior a las coaguladas por cambio de $\mathrm{pH}$ hasta el isoeléctrico, lo que hizo pensar a Lange et al. $(59,60)$ que la adición de sal provocaba la aparición de un nuevo tipo de fuerza de interacción, no predicha por la teoría clásica de DLVO. Israelachvili y Horn $(23,24,26)$ encontraron evidencias experimentales de que existían fuerzas de interacción entre las partículas y el líquido (fuerzas de hidratación, en el caso del agua) de corto alcance y fuertemente repulsivas. Velamakanni y Lange (59) especularon que se podía originar una fuerza repulsiva de corto alcance a partir de la deshidratación de iones contrarios alrededor de la superficie de las partículas cuando la fuerza iónica es alta. La manipulación de las propiedades coloi-

\section{Suspensión defloculada}

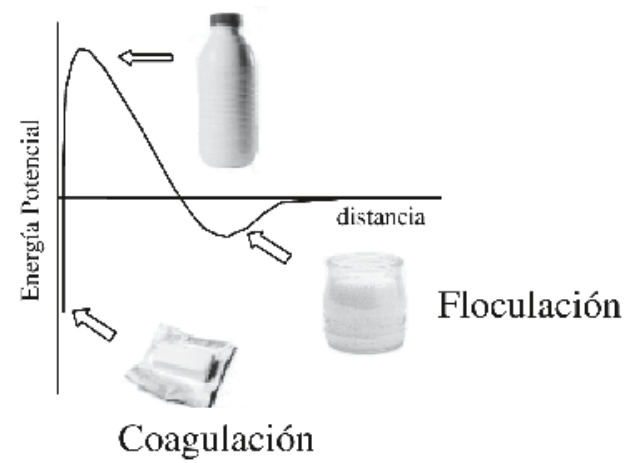

Fig. 4.- Representación de la energía de interacción entre partículas en función de la distancia entre ellas.
A

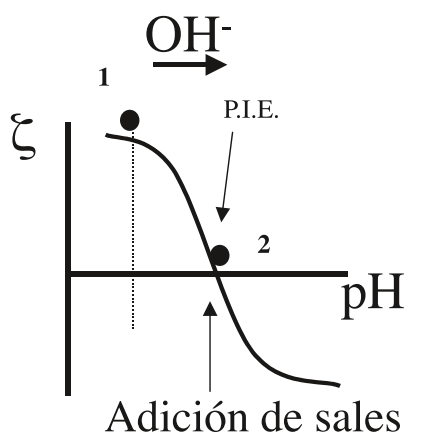

B

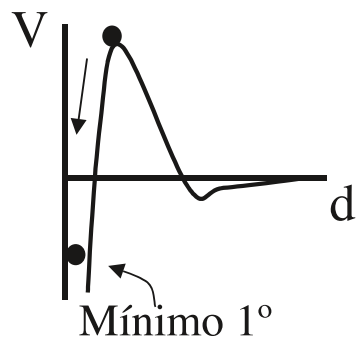

Fig. 5.- Conformado por coagulación directa: a) coagulación por cambio de pH hasta el punto isoeléctrico, b) representación de la coagulación mediante la curva de energía de interacción en función de la distancia.
A

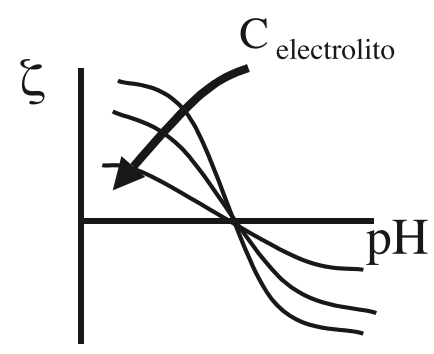

B

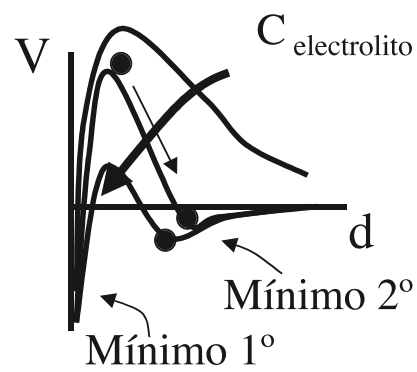

Fig. 6.- Desarrollo de fuerzas repulsivas de corto alcance mediante la adición de sales: a) efecto de la concentración de sal en el potencial zeta, b) representación de la coagulación mediante la curva de energía de interacción en función de la distancia.

dales de una suspensión ha permitido a dichos investigadores desarrollar una red debilmente atractiva entre partículas desplazando el mínimo secundario a menores distancias de separación en la curva de energía potencial (59-61). De esta forma, a partir de una suspensión estable con alta concentración en sólidos (40-50\% vol) la adición de sales provoca un aumento brusco de viscosidad que permite obtener una pasta de alta plasticidad fácilmente moldeable. En la curva de potencial zeta frente a $\mathrm{pH}$ (figura 6.a) el punto isoeléctrico no varía, pero los potenciales zeta son menores al aumentar la concentración de sales. En la curva de energía potencial (figura 6.b) esto se traduce en un mínimo secundario que, al aumentar la concentración de sales, 
se aproxima a la distancia de contacto. No obstante, la suspensión así preparada precisa de una etapa de conformado posterior, bien por filtración bajo presión, o bien por centrifugación.

En los dos métodos expuestos se parte de una suspensión estabilizada electrostáticamente. Sin embargo, cuando se usan suspensiones defloculadas estéricamente con polímeros, es también posible provocar una desestabilización térmica reversible del mismo. Así, se ha propuesto un nuevo método de conformado (temperature induced forming, TIF) basado en la creación de una red de partículas termoreversible que se consigue mediante una buena combinación de disolvente y dispersante polimérico que, al bajar la temperatura, empeore la solubilidad del polímero y permita la floculación por puentes poliméricos, lo que genera un aumento brusco de viscosidad (62). Asimismo, se ha descrito la posibilidad de obtener piezas a partir de suspensiones acuosas mediante formación de puentes polímericos entre las moléculas de aglomerante al cambiar la temperatura (63). La figura 7 ilustra la formación de puentes poliméricos que producen una red atractiva de partículas (figura 7.A), así como el efecto en el potencial de repulsión, en cuyo caso las partículas caen en un mínimo de floculación (figura 7.B).

Estos procesos demuestran el espectacular avance experimentado en la ciencia del procesamiento cerámico que ha sabido incorporar los conocimientos aportados por la ciencia de coloides y la reología.

Por otra parte, el conocimiento más exhaustivo de los aditivos de procesamiento ha permitido desarrollar recientemente otros procesos de conformado casi neto mediante el empleo de aglomerantes y gelificantes que provocan la congelación de la estructura de la suspensión previamente estabilizada con resistencias muy superiores a las alcanzadas en ausencia de dichos aditivos. Esto ha provocado un creciente interés por las técnicas de polimerización/gelificación que se describen a continuación.

\subsection{Métodos de gelificación.}

El empleo de aditivos orgánicos es práctica habitual en el procesamiento cerámico, desde los métodos de prensado hasta los de moldeo por inyección, pasando por los de colaje y extrusión $(3,64,65)$. Una de las tendencias más actuales en el conformado es provocar la gelificación de una suspensión una vez que se ha introducido en un molde de forma tan compleja como permita su elaboración. Estas técnicas aúnan la mejor comprensión de la coloidequímica de suspensiones cerámicas con la ciencia y tecnología de polímeros.

La gelificación de los aditivos orgánicos se puede conseguir por dos vías (66): 1) polimerización por reacción química de monómeros, y 2) gelificación térmica, en la que un polímero pasa de ser fluido a ser una sustancia vítrea de elevada rigidez por cambio de temperatura si se atraviesa la temperatura de transición vítrea (Tg). El primer caso es la base del proceso de colaje con gelificación (gelcasting), mientras que el segundo es clave en procesos de consolidación térmica, como el moldeo por inyección. A continuación se describen brevemente ambos procesos.

El proceso de gelcasting, desarrollado por Janney y Omatete (67-69), consiste en la preparación de una suspensión estable del polvo cerámico en un disolvente con los defloculantes necesarios para la estabilización, sobre la cual se añaden monómeros que polimerizan para formar un gel de alta resistencia. La disolución de monómeros proporciona una baja viscosidad, lo
A

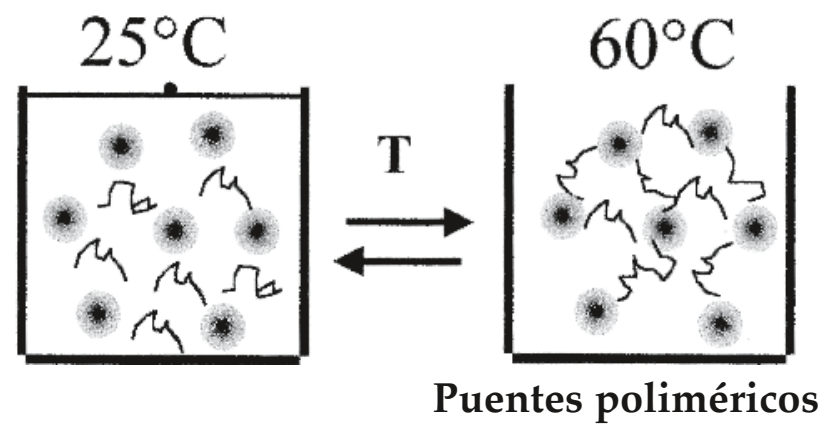

B

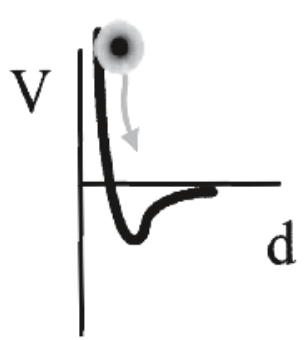

Fig. 7.- Formación de puentes poliméricos en el proceso de floculación inducida por temperatura en medio acuoso (A) y representación de la floculación en mínimo secundario en suspensiones estabilizadas con polímeros (B).

que facilita su uso como vehículo de transporte del polvo. La polimerización provoca la formación de un gel entrecruzado polímero/disolvente que inmoviliza a las partículas en la geometría deseada tras su vertido en un molde. La concentración típica de polímero es de 10-20\% vol, algo inferior a la utilizada en el moldeo por inyección convencional. Normalmente se usan contenidos en sólidos de 50-60\% vol. Además de los aglomerantes monoméricos, se precisa la acción de iniciadores/ catalizadores para provocar la polimerización de aquellos. Aunque en un principio el proceso se ideó para disolventes orgánicos, posteriormente se extendió a medios acuosos utilizando acrilamidas (69-70). Una gran ventaja es que se pueden emplear moldes de materiales muy diversos y poco costosos, como son metales, vidrio, plásticos, ceras, etc. Además de permitir la obtención de piezas de forma compleja, la elevada resistencia del gel formado permite su fácil mecanizado en verde para obtener formas aún más complejas. Sin embargo, las concentraciones de aditivo empleadas son relativamente altas (4-10\% vol), lo que exige un ciclo térmico específico para la completa eliminación de los orgánicos, etapa crítica en la cual se pueden originar defectos tales como erupciones, deformación de la pieza o aparición de grietas.

La gelificación térmica de un polímero constituye la base del moldeo por inyección (71-73). Este proceso es el tradicionalmente empleado en la fabricación de piezas de geometría compleja. Normalmente, se parte de una mezcla de polvo cerámico en un polímero fundido que se inyecta en la cavidad de un molde. Se pueden dar dos casos dependiendo de la naturaleza del polímero (74): 1) polímero termoestable, que funde a una temperatura superior a la $\mathrm{Tg}$ y 2) polímero termoendurecedor, que por encima de la $\mathrm{Tg}$ se hace rígido. En el primer caso, la 
mezcla se prepara en caliente y se inyecta en un molde frío, mientras que en el segundo la mezcla a temperatura ambiente gelifica al inyectarla en un molde caliente. En el moldeo por inyección convencional la elevada concentración de polímero (20-50\% vol) proporciona una viscosidad muy alta, por lo que la presión de inyección es también alta (10-150 MPa). Un avance decisivo en la reducción de costos de producción lo supuso el desarrollo de los procesos de moldeo por inyección a baja presión (LPIM), en el que los polímeros se sustituían por ceras, y en concentraciones más bajas (10-20\% vol). Esto supone una reducción importante en la viscosidad, de forma que basta una presión inferior a $1 \mathrm{MPa}$ para llevar a cabo la inyección (7577).

Un avance posterior fue el desarrollo de este método para la gelificación de suspensiones acuosas. El proceso de LPIM tiene su origen en el comportamiento de gelificación de la metilcelulosa y sus derivados, que gelifican por encima de la $\mathrm{Tg}$ (78-79). Por lo tanto, la gelificación ocurre al inyectar una mezcla fría en un molde caliente. Esta propiedad de gelificación térmica fue utilizada por primera vez en 1976 por Rivers (80) para el moldeo por inyección de polvos.

Fanelli et al. (81) propusieron en 1988 el uso de derivados del agar para el conformado por LPIM de suspensiones acuosas calentadas por encima de la Tg e inyectadas en un molde refrigerado, de forma que se producía la gelificación inmediata del aglomerante. En este proceso se utilizan suspensiones acuosas con contenidos en sólidos de 40-50\% vol previamente defloculadas sobre las que se añade una baja concentración de gelificante (2-3\% vol). Las ventajas del proceso son varias. Por una parte, la concentración de aditivo es muy baja, lo que permite obtener una pieza que, tras el secado, se puede someter al ciclo de sinterización sin necesidad de un ciclo específico de extracción de orgánicos (81-84). Por otra parte, se pueden obtener piezas de forma casi final, bien por vertido, bien por inyección. Además, los derivados del agar, y especialmente la agarosa, proporcionan una resistencia en verde muy elevada, por lo que la pieza en verde puede ser fácilmente manipulada y mecanizada.

Las mayores limitaciones de este proceso se refieren al espesor de la pieza a conformar, a la posibilidad de conformar piezas huecas y al elevado costo de los aditivos empleados hasta ahora. Por lo que se refiere al espesor, la gelificación se produce por contacto con un molde frío, lo que siginifica que si la pieza es voluminosa la transmisión de frío hacia el interior es lenta, lo que resultará en una parte externa consolidada mientras que el interior está todavía líquido. De igual forma, resulta dificil frenar la gelificación en el espesor deseado, por lo que aún no se ha descrito el empleo de esta técnica para el conformado de piezas huecas. Sin embargo, la mayor limitación para su aplicación industrial es, como se señalaba arriba, el costo desproporcionadamente alto de los aditivos. La agarosa es el aditivo que mayor resistencia de gel proporciona $\left(>1200 \mathrm{~g} / \mathrm{cm}^{2}\right)$, pero $\mathrm{su}$ costo puede superar $250 \$ / \mathrm{Kg}$. Dado el enorme potencial de esta técnica de conformado casi final, en la actualidad numerosos grupos de investigación están centrando sus esfuerzos en el desarrollo y control de aditivos más baratos, como el agar (85) y otros polisacáridos. Así, se ha descrito ya el uso de almidones (86), proteinas y gelatinas (87), pero las concentraciones de aditivo son mucho más elevadas y la resistencia de gel notoriamente más baja, lo que redunda en tiempos de proceso mayores, consistencia en verde inferior $y$, a menudo, necesidad de un ciclo de extracción de orgánicos.

\section{CONCLUSIONES}

En los últimos años se han desarrollado numerosas técnicas de conformado a partir de suspensiones. Esto ha sido posible gracias a la mayor preocupación de los ceramistas por incorporar los avances de la ciencia de los coloides y de las técnicas reométricas en el control de los procesos cerámicos. A la vista de los trabajos publicados en los últimos años se puede decir que hay dos grandes preocupaciones en el área del conformado de piezas. Por una parte, encontrar métodos de conformado versátiles y de bajo costo capaces de producir piezas de elevada complejidad, con el fin de eliminar o reducir la etapa de mecanizado. Paralelamente, se ha potenciado la búsqueda de nuevos aditivos, más eficaces y económicos que, por una parte, ayuden a reducir los problemas medioambientales y, por otra, proporcionen piezas con mayor resistencia en verde. Además, se fomenta el uso de agua en procesos que tradicionalmente no la usaban, como es el caso del moldeo por inyección, o el colaje con gelificación, e incluso procesos de conformado de sustratos y recubrimientos, como el colaje en cinta o la deposición electroforética. Los procesos de conformado de piezas de forma casi final utilizan suspensiones de elevada concentración en sólidos (hasta 60\% vol), lo que supone una reducción importante de la cantidad de agua a eliminar durante el conformado y el secado. Todo esto es posible gracias a una cada vez más minuciosa caracterización de los parámetros reológicos de las suspensiones. De todos los procesos mencionados en este trabajo, el colaje con presión es ya una realidad en distintos sectores de producción, mientras que el proceso LPIM despunta como uno de los de mayor potencial para la fabricación de piezas pequeñas de geometría compleja.

\section{AGRADECIMIENTOS}

Este trabajo ha sido financiado por un proyecto dotado por la CICYT (MAT97-0676).

\section{BIBLIOGRAFÍA}

1. G.Y. Onoda, L.L. Hench (eds). "Ceramic Processing Before Firing", John Wiley \& Sons, New York (EEUU) 1978.

2. F.F.Y. Wang (ed) "Treatise on Materials Science and Technology, vol 9: Ceramic Fabrication Processes". Ed.. Academic Press, New York (EEUU) 1978.

3. J.S. Reed. "Introduction to the Principles of Ceramic Processing" $2^{\text {nd }}$ ed, John Wiley \& Sons, New York (EEUU), 1995.

4. F.F. Lange. "Powder Processing Science and Technology for Increased Reliability", J. Am. Ceram. Soc., 72 [1] 3-15 (1989).

5. C.J. Brinker, D.E. Clark, D.R. Ulrich (eds). "Better Ceramics through Chemistry", Mat. Res. Soc. Proc., vol 32, North-Holland, New York (EEUU) 1984.

6. L.L. Hench, D.R. Ulrich (eds). "Ultrastructure Processing of Ceramics, Glasses and Composites", John Wiley \& Sons, New York (EEUU) 1984.

7. D.E. Niesz. "Advanced Powder Technology", Ceram. Eng. Sci. Proc. 5 [3-4] 159-67 (1984).

8. D.W. Richerson. "Modern Ceramic Engineering. Properties, Processing and Use in Design" $2^{\text {nd }}$ ed, Marcel Dekker, Inc., New York, 1992.

9. W. Bunk, H. Hausner (eds). "Ceramic Materials and Components for Engines", Verlag Inc., Köln (Alemania) 1986.

10. R.A. Terpstra, P.P.A.C. Pex, A.H. De Vries (eds). "Ceramic Processing". Chapman \& Hall, London (UK) 1995.

11. "Shaping of Advanced Ceramics", Refereed Reports. J. Eur. Ceram. Soc. 17 [2-3] 1997.

12. W. Jaschinski, A. Nagel. "Possibilities and Limits in the Shaping of Ceramic Powders", Interceram 42 [3] 135-39 (1993).

13. L.M. Sheppard. "Fabrication of Ceramics: The Challenge Continues", Am Ceram. Soc. Bull. 68 [10] 1815-20 (1989).

14. J.A. Pask. "Ceramic Processing. A Ceramic Science”. Am. Ceram. Soc. Bull. 58, 1163 (1979).

15. P.F. Messer. "Ceramic Processing. A Systematic Approach", Trans. Br. Ceram. Soc. 82 [6] 190-92 (1983).

16. I.A. Aksay, F.F. Lange, B.I. Davis. “Uniformity of $\mathrm{Al}_{2} \mathrm{O}_{3} / \mathrm{ZrO}_{2}$ Composites by Colloidal Filtration", J. Am. Ceram. Soc. 60 [10] C190-C192 (1983).

17. I.A. Aksay, C.H. Schilling. "Colloidal Filtration Route to Uniform Microstructure", pp. 483-91 en "Ultrastructure Processing of Ceramics, Glasses and Composites". Ed. L.L. Hench, D.R. Ulrich. John Wiley \& Sons, New York (EEUU) 1984. 
18. C.W. Hong. "From Long-Range Interactions to Solid-Body Contact Between Colloidal Surfaces during Forming" J. Eur. Ceram. Soc. 18, 2159-67 (1998)

19. R. Moreno, C.A. Gutiérrez. "Tape Casting Technology: Role of Particle Pair Potentials", pp. 611-22 en "9 $9^{\text {th }}$. CIMTEC-World Ceramic Congress. Ceramics Getting into the 2000`s-Part B". Ed. P. Vincenzini, Techna Srl., Faenza (Italia) 1999.

20. J.W. Goodwin. "Rheology of Ceramic Materials", Am. Ceram. Soc. Bull. 69 [10] 1694-98 (1990)

21. L. Bergström. "Rheology of Concentrated Suspensions", pp. 193-244 en "Surface and Colloid Chemistry in Advanced Ceramics Processing". Ed. R.J. Pugh, L. Bergström. Marcel Dekker, Inc, New York (EEUU) 1994.

22. C.A. Gutiérrez, A.J. Sánchez-Herencia, R. Moreno. “¿Plástico o Pseudoplástico? Métodos de Determinación y Análisis del Punto de Fluidez de Suspensiones Industriales", Bol. Soc. Esp. Ceram. Vidr. 39 [1] 105-117 (2000).

23. R.G. Horn. "Surface Forces and Their Action in Ceramic Materials", J. Am. Ceram. Soc., 73 [5] 1117-1135 (1990).

24. J.N. Israelachvili, G.E. Adams. "Measurement of Forces between Two Mica Surfaces in Aqueous Electrolyte Solutions in the Range 0-1000 nm", J. Chem. Soc. Farady Trans. 74 [1] 975-1001 (1978).

25. R. J. Hunter. "Foundations of Colloidal Science". Oxford Science Pub., Oxford (UK) 1986

26. J.N. Israelachvili. "Intermolecular and Surface Forces" $2^{\text {nd }}$ ed. Academic Press, London (UK) 1992.

27. R.J. Pugh. "Dispersion and Stability of Ceramic Powders in Liquids", pp. 127-92 en "Surface and Colloid Chemistry in Advanced Ceramics Processing". Ed. R.J. Pugh, L. Bergström. Marcel Dekker, Inc, New York (EEUU) 1994.

28. R. Moreno. "The Role of Slip Additives in Tape casting Technology. I: Solvents and Dispersants". Am. Ceram. Soc. Bull. 71 [10] 1521-31 (1992).

29. H.M. Jang. "Colloid interface Science for Ceramic Powder Processing", pp. 157-213 en "Chemical Processing of Ceramics". Ed. B.I. Lee, J.A. Pope. Marcel Dekker, New York (EEUU) 1994.

30. C. Greskovich. "Milling", pp. 15-33 en "Treatise on Materials Science and Technology, vol 9: Ceramic Fabrication Processes". Ed. F.F.Y. Wang. Academic Press, New York (EEUU) 1978.

31. D.S. Adcock, I.C. McDowall. "The Mechanism of filter Pressing and Slip Casting". J. Am. Ceram. Soc. 40 [10] 355-62 (1957)

32. F.M. Tiller, C.D. Tsai. "Theory of Filtration of Ceramics: I. Slip Casting". J. Am. Ceram. Soc. 69 [12] 882-87 (1986).

33. R. Moreno, J.S. Moya, J. Requena. "Colaje de Oxidos Cerámicos. I. Fundamentos" Bol. Soc. Esp. Ceram. Vidr. 24 [3] 165-71 (1985)

34. J.E. Funk, D.R. Dinger. "Modeling the Slip Casting Process". Ceram. Eng. Sci. Proc. 11 [3-4]217-27 (1990).

35. R.E. Mistler, D.J. Shanefield, R.B. Runk. "Tape Casting of ceramics", pp. 411-47 "Ceramic Processing Before Firing". Ed. G.Y. Onoda, L.L. Hench. John Wiley \& Sons, New York (EEUU) 1978.

36. R. Moreno, J. Requena. "Introducción al Colaje de Cinta". Bol. Soc. Esp. Ceram. Vidr. 31 [2] 99-108 (1992).

37. P. Sarkar, P.S. Nicholson. “Electrophoretic Deposition (EPD): Mechanisms, Kinetics, and Application to ceramics". J. Am. Ceram. Soc. 79 [8] 1987-2002 (1996).

38. B. Ferrari, R. Moreno. “Conformado de Materiales Cerámicos por Electroforesis en Medios Acuosos". Bol. Soc. Esp. Ceram. Vidr. 38 [5] 369-81 (1998).

39. L. Bergström. "Colloidal processing of Ceramics-Technology and Science", pp. 34148 en "Ceramic Transactions, vol 51: Ceramic Processing Science and Technology". Ed. H. Hausner, G.L. Messing, S. Hirano. The Am. Ceram. Soc., Westerville, OH (EEUU) 1995.

40. E.F. Adams. "Slip Cast Ceramics", pp. 145-84 en "High Temperature Oxides, part IV". Ed. A.M. Alper. Academic Press, New York (EEUU) 1971

41. E.G. Blanchard. "Pressure Casting Improves Productivity". Am. Ceram. Soc. Bull. 67 [10] 1680-83 (1988).

42. H. Pfuhl. "Development of the Tableware and Sanitaryware Industry from 19501990". Interceram 41 [4] 234-36 (1992).

43. B. Leach, H. Wheeler, B. Lynne. "Analysis of Pressure Casting Rates". Am. Ceram. Soc. Bull. 75 [8] 49-51 (1996)

44. F.F. Lange, K.T. Miller. "Pressure Filtration: Consolidation Kinetics and Mechanics". Am. Ceram. Soc. Bull. 68 [10] 1498-1504 (1987).

45. Report, "Pressure Casting-An Established Production Process". Interceram 39 [6] 45-47 (1990).

46. K. Bridger, M. Massuda. “Principles of Slip Casting/Pressure Filtration”, pp. 507-19 en "Ceramic Transactions, vol 12: Ceramic Powder Science III". Ed. G.L. Messing, S. Hirano, H. Hausner. The American Ceramic Society, Inc., Westerville, $\mathrm{OH}$ (EEUU) 1990.

47. H. Nienburg, F. Harbach. "Pressure Filtration of Fine Ceramic Suspensions", pp. 321-27 en "Ceramic transactions, vol 22: Ceramic Powder Science, IV". Ed. S. Hirano, G.L. Messing, H. Hausner. The American Ceramic Society, Westerville, $\mathrm{OH}$ (EEUU) 1992.

48. E.G. Blanchard. "Pressure Slip casting”. Ceram. Eng. Sci. Proc. 11 [11-12] 1797-1803 (1996).

49. E. Beylier, R.L. Pober, M.J. Cima. "Centrifugal-Casting of Ceramic Components", pp. 529-36 en "Ceramic Transactions, vol 12: Ceramic Powder Science III". Ed. G.L. Messing, S. Hirano, H. Hausner. The American Ceramic Society, Inc., Westerville, OH (EEUU) 1990

50. W. Huisman, T. Graule, L.J. Gauckler. "Centrifugal Slip Casting of Zirconia (Y-TZP)". J. Eur. Ceram. Soc. 13 [1]33-39 (1994).

51. I.J. Chabinsky. "A Microwave Energy Enhanced Slip Casting System". Interceram 3[1] 38-39 (1987)

52. J.G.P. Binner. "The Effect of Raising Temperature Using Microwaves on the Slip Casting of Alumina Based Ceramics". Br. Ceram. Trans. J. 91 [1] 48-51 (1992).

53. R. Moreno, A. Salomoni, I. Stamenkovic. "Influence of Slip Rheology on Pressure
Casting of $\mathrm{Al}_{2} \mathrm{O}_{3}{ }^{\prime \prime}$. J. Eur. Ceram. Soc. 17 [2-3] 327-31 (1997).

54. R. Moreno, A. Salomoni, I. Stamenkovic, S.M. Castanho. "Colloidal Filtration of Silicon Nitride Aqueous Slips. Part II: Slip Casting and Pressure Casting Performance". J. Eur. Ceram. Soc. 19 [1] 49-59 (1999).

55. J. Holly, D. Hampton, S.B. Savage, R.A.L. Drew. "Experimental Analysis of FineParticle Migration during Ceramic Filtration Processes". J. Am. Ceram. Soc. 75 [10] 2726-32 (1992).

56. M.I. Nieto, R. Moreno, A. Salomoni, I. Stamenkovic. “Aqueous Pressure Casting Improves Manufacturing of SiC Parts". Am. Ceram. Soc. Bull. 77 [11] $62-66$ (1998).

57. T.J. Graule, F.H. Baader, L.J. Gauckler. "Shaping of Ceramic Green Compacts Direct from Suspensions by Enzyme Catalyzed Reactions". Cfi/Ver. DKG 71, 317-23 (1994).

58. L.J. Gauckler, F.H. Baader, T.J. Graule. “Direct coagulation Casting (DCC); Foundamentals of a New Forming Process for Ceramics", pp. 457-62 en "Ceramic Transactions, vol 51: Ceramic Processing Science and Technology". Ed. H. Hausner, G.L. Messing, S. Hirano. The Am. Ceram. Soc., Westerville, OH (EEUU) 1995.

59. B.V Velamakanni, J.C. Chang, F.F. Lange, D.S. Pearson. “New Method for Efficient Colloidal Particle Packing via Modulation of Repulsive Lubricating Hydration Forces". Langmuir 6 [7] 1323-25 (1990).

60. F.F. Lange. "New Interparticle Potential. Paradigm Form Advanced Powder Processing", pp. 185-201 en "Ceramic transactions, vol 22: Ceramic Powder Science, IV". Ed. S. Hirano, G.L. Messing, H. Hausner. The American Ceramic Society, Westerville, OH (EEUU) 1992.

61. B.V Velamakanni, F.F. Lange, F.W. Zok, D.S. Pearson. “Influence of Interparticle Forces on the Rheological Behavior of Pressure-Consolidated Alumina Particle Slurries". J. Am. Ceram. Soc. 77 [1] 216-20 (1994).

62. L. Bergström, S. Sjöström. “Temperature Induced Gelation of Concentrated Ceramic Suspensions: Rheological Properties". J. Eur. Ceram. Soc. 19, 2117-23 (1999).

63. N.S. Bell, L. Wang, W.M. Sigmund, F. Aldinger. "Temperature Induced Forming: Application of Bridging Flocculation to Near-Net Shaping Production of Ceramic Parts". Z. Metallkd. 90 [6] 388-92 (1999).

64. R. Moreno. "The Role of Slip Additives in Tape casting Technology. II: Binders and Plasticizers". Am. Ceram. Soc. Bull. 72 [11] 1747-57 (1992).

65. D.J. Shanefield. "Organic Additives and Ceramic Processing", $2^{\text {nd }}$ ed. Kluwer Ac. Pub., Norwell, MA (EEUU) 1996

66. J.R. Fried. "Polymer Science and Technology". Prentice Hall PTR, New Jersey (EEUU) 1995.

67. M.A. Janney. "Method for Forming Ceramic Powders into complex Shapes". U.S Pat $4894194,1990$.

68. O.O. Omatete, .M.A. Janney, R.A. Sthrelow. “Gelcasting-A New Ceramic Forming Process". Am. Ceram. Soc. Bull. 70 [10] 1641-49 (1991).

69. O.O. Omatete, .M.A. Janney, S.D. Nunn. “Gelcasting: From Laboratory Developmen toward Industrial Production". J. Eur. Ceram. Soc. 17 [2-3] 407-13 (1997).

70. M.A. Janney, O.O. Omatete. "Method for Molding Ceramic Powders using WaterBased Gel Casting". U.S. Pat. 5028362, 1991.

71. A. Odriozola, I. Ochoa, M. Gutiérrez. "Moldeo por Inyección de Cerámicas". Bol. Soc. Esp. Ceram. Vidr. 33 [5] 267-71 (1994).

72. R.M. German, K.F. Hens. "Key Issues in Powder Injection Molding". Am. Ceram. Soc.Bull. 70 [8] 1294-1302 (1991).

73. B.C. Mutsuddy, R.G. Ford. "Ceramic Injection Molding". Chapman \& Hall Cambridge (EEUU) 1995.

74. M.J. Edirisinghe, J.R.G. Evans. "Review: Fabrication of Engineering Ceramics by Injection Moulding. I: Materials Selection". Int. J. High Technology Ceramics 2, 1-31 (1986).

75. M. Peltsman. "Low Pressure Injection Molding and Mold Design". Metal Powder Report 41, 367-69 (1985)

76. J.A. Mangels. "Low-Pressure Injection Molding”. Am. Ceram. Soc. Bull. 73 [5] 37-41 (1994).

77. S. Novak, K. Vidovic, M. Sajko, T. Kosmac. "Surface Modification of Alumina Powder for LPIM". J. Eur. Ceram. Soc. 17 [2-3] 217-24 (1997).

78. N. Sarkar, G.K. Greminger. “Methylcellulose Polymers as Multifunctional Processing Aids in Ceramics". Am. Ceram. Soc. Bull. 61[11] 1280-84 (1983).

79. J.E. Schuetz. "Methylcellulose Polymers as Binders for Extrusion of Ceramics". Am. Ceram. Soc. Bull. 65 [12] 1556-59 (1986).

80. R.D. Rivers. U.S. Pat No $4113480,1978$.

81. A.J. Fanelli, R.D. Silvers, W.S. Frei, J.V. Burlew, G.B. Marsh. “New Aqueous Injection Molding Process for Ceramic Powders". J. Am. Ceram. Soc. 72 [10] 1833-36 (1989).

82. Z.S. Rak, F.W. Hamburg. "Manufacturinf of Alumina Products by Low and High Pressure Injection Moulding". Ceramica Acta 7 [2-3] 5-13 (1995).

83. Z.S. Rak, P.J. van Tiborg. "Aqueous Injection Moulding Process", pp. 409-13 en "Euro-Ceramics II, vol 1: Basic Science and Processing of Ceramics". Ed. G. Ziegler, H. Hausner. DKG, Berlin (Alemania) 1991

84. A.J. Millám, M. Bravo, R. Moreno, M.I. Nieto. “Moldeo por Inyección a Baja Presión de Suspensiones Cerámicas". Bol. Soc. Esp. Ceram. Vidr. 38 [2] 93-99 (1999).

85. T. Zhang, S. Blackburn, J. Bridgwater. "Properties of Ceramic Suspensions for Injection Moulding Based on Agar Binders". Br. Ceram. Trans. 96 [6] 229-33 (1994).

86. O. Lyckfeldt,J.M.F. Ferreira. "Processing of Porous Ceramics of Starch Consolidation". J. Eur. Ceram. Soc. 18, 131-40 (1998)

87. Y. Chen, Z. Xie, J. Yang, Y. Huang. "Alumina Casting Based on Gelation of Gelatine". J. Eur. Ceram. Soc. 19, $271-75$ (1999).

Recibido: 22-11-.99

Aceptado: 20-1-00 